\title{
The Numerical Investigation on Bubble Interaction Dynamics in Hydrodynamic Cavitation
}

\author{
Liang LV*, Xu LUO**, Hongxia ZHANG***, Bing CUI****, Lihai CHEN***** \\ *Chengde Petroleum College, Chengde067000,China,_E-mail:1vl_5687289@163.com \\ **Chengdu University of Technology, Chengdu 610059,China,E-mail: lxshisan@163.com \\ ***Chengde Petroleum College, Chengde 067000,China,E-mail:13633146517@126.com \\ ****Chengde Petroleum College, Chengde 067000, China,E-mail: cuibing93@163.com \\ *****Chengde Petroleum College, Chengde 067000, China, E-mail: chenlh_19931099334@163.com \\ crossref http://dx.doi.org/10.5755/j02.mech.26187
}

\section{Nomenclature}

\section{Roman letters}

$R$ is instantaneous bubble radius; $\dot{R}$ is first derivative of the instantaneous bubble radius; $\ddot{R}$ is second derivative of the instantaneous bubble radius; $R_{0}$ is initial bubble radius; $c$ is speed of sound in the liquid; $P_{0}$ is ambient pressure; $d_{i}$ is distance to its neighbor bubble with radius; $R_{i} ; S$ is coupling strength of bubbles; $p_{t}$ is instantaneous liquid pressure; $p_{v}$ is vapor pressure under operating temperature; $p_{2}$ is recovery pressure in the pipe; $v_{0}$ is fluid velocity at the orifice; $v_{p}$ is fluid velocity at pipe; $L$ is length scale of eddy; $d_{p}$ is pipe diameter; $C_{i}$ is cavitation number; $d_{0}$ is orifice diameter; $T_{\infty}$ is ambient temperature in the liquid; $p(t)$ is internal pressure as a function of time; $V(t)$ is bubble volume as a function of time; $p_{\text {min }}$ is minimum internal pressure; $V_{\max }$ is maximum bubble volume; $R_{\max }$ is maximum bubble radius; $R_{\min }$ is minimum bubble radius.

Greek letters

$\gamma$ is ratio of specific heats of gas; $\mu$ is viscosity of the liquid; $\rho$ is density of the liquid; $\sigma$ is surface tension coefficient of the liquid; $\tau$ is pressure recovery time; $\beta$ is orifice-topipe-diameter.

\section{Introduction}

Cavitation has attracted the attentions of many researchers due to its application in many fields. The dynamics of singe bubble has been investigated theoretically, experimentally and numerically [1-8]. In many situations, the bubbles are sufficiently close to form a cluster. Bubble-bubble interactions have great influences on cavitation applications, which include not only traditional fields such as pressure fluctuation in hydrodynamic machinery [9], cavitation flowing [10] and cavitation erosion in silt-laden liquid [11], but also ultrasonic cleaning [12], sonoluminescence [13], sonochemistry [14], water treatment [15], and so on.

In the experimental studies on the bubble-bubble interactions, the bubbles were usually generated by the methods of electric discharge, optical breakdown (laser) and pressure impulse at fixed positions, and the bubble dynamics were generally observed by high-speed photography [1621]. For two-bubble interactions, four types of bubble oscillation behaviors were observed, namely, jetting toward each other, jetting away from each other, bubble coalescence and "catapult" effect, which were controlled by three parameters, i.e., the difference time of the bubbles generation, bubble- bubble dimensionless distance and relative sizes [16-18]. The three/four-bubble interactions were more complicated which were affected by many factors such as bubble generation positions, bubble sizes and bubble phase. Multi-bubbles oscillation period increases with bubbles coalescence but change little without coalescence [19]. An experiment was designed in which 37 bubbles were generated simultaneously under the action of acoustic wave at hydrophobic microcavity etched on a silicon plate in Ref. [20]. Authors found the expansion and collapse of the inner bubbles are delayed due to the affects of the outer bubbles. Following the original idea in Ref. [20], the interacting two bubble clouds and sonochemical production were investigated experimentally and theoretically [21].

The interactions of two bubbles and multibubble have been carried out theoretically and numerically. The mutual force between two bubbles was called the secondary Bjerknes force which caused the bubbles to either repel or attract each other [6]. The changes of the sign of the secondary Bjerknes force due to the variation of the transition frequencies of two-bubble system were investigated in Ref. [22]. Sugita and Sugiura [23] investigated the bifurcation of two spherical bubbles by the method of nonlinear model analysis. Valier-Brasier and Conoir [24] analyzed the resonant acoustic scattering of two spherical bubbles using the addition theorem and spherical harmonics expansions. In addition to the good studies of two bubble model and their dynamics, the researchers have investigated the dynamics of the bubble cluster. There were two complementary approaches to investigate bubble clouds dynamics. One was that bubble-liquid mixture was continuous, and the macroscopic motion of a bubble cluster was analyzed. The other was that the microscopic motion of single bubble in the cluster was investigated [25]. The studies of first group were mostly focused on the linearized dynamics of the bubble clusters. In the paper of Fuster and Colonius [26], based on volume-averaged equations taking bubble-bubble interactions into account, a new Rayleigh-Plesset equation was proposed to predict the dynamics of a bubble cluster. Taking advantage of this equation acoustic waves propagation theory in bubbly liquid was modified account for bubble-bubble interactions [27]. The second group focused on the interactions between bubbles in a cluster. In the work of An [13], the multibubble sonoluminescence in a small bubble cluster was investigated numerically using a new model assuming all bubbles arrayed homogenously. A bubble cluster model describing high number density of microbubbles and large size of bubble cluster was proposed in Ref. [28]. The 
maximum characteristic size of a bubble cluster reached 0.5 $\mathrm{mm}$ and the coupling strength could be as large as $10^{6} \mathrm{~m}^{-1}$. This model could accurately predict the destruction of encapsulated bubbles under the effects of bubble-bubble interactions [29].

Depending on the formation condition of local pressure, there were two kinds of cavitation, namely, ultrasonic cavitation and hydrodynamic cavitation. The interactions between ultrasonic cavitation bubbles have been investigated theoretically a lot and abundant results have been obtained [6, 13, 21-29]. The dynamics of two bubbles in hydrodynamic cavitation were revealed in Refs. [30, 31]. They found that there was a lag during the larger bubble oscillations under the exist of a smaller bubble, and lag became more and more obvious as time went on. Nevertheless, there were few studies in regard to the dynamics of bubble cluster in hydrodynamic cavitation. Therefore, in present paper we consider a new bubble cluster model based on the original idea in Ref. [28] accounting for bubble-bubble interactions in hydrodynamic cavitation. The bubble dynamics are investigated theoretically and numerically within a wide range of parameter zone (e.g. the bubble number in a cluster). The sections of present paper are organized as follows. Section 2 introduces the full model of a bubble cluster in hydrodynamic cavitation and numerical methods. Section 3 discusses the influences of parameters on the dynamics of a bubble cluster. Section 4 summaries the main findings of the present paper.

\section{Mathematical model and numerical method}

The geometry of orifice plate is shown in Fig. 1 . The radial motion of a bubble is described by modified Rayleigh-Plesset equation which considers the effects of the compressibility, viscosity and surface tension of the liquid [32]. Some following assumptions are made to establish bubble interaction dynamics in hydrodynamic cavitation. (1) The bubble keeps spherical and their centers are fixed. (2) Bubbles are spatially homogeneously distributed and oscillate in the same manner. (3) There are no gas diffusion and heat transfer during bubble expansion and collapse. (4) The bubble expansion is isothermal and its compression is adiabatic. The equation using in present model can be written as Eq. (1) [28, 29, 33].

$$
\begin{aligned}
& \rho R \ddot{R}\left(1-\frac{\dot{R}}{c}\right)+\frac{3}{2} \rho \dot{R}^{2}\left(1-\frac{1}{3} \frac{\dot{R}}{c}\right) \\
&=\left(1+\frac{\dot{R}}{c}\right) p_{\text {diff }}(R, t)+\frac{R}{c} \frac{d p_{\text {diff }}(R, t)}{d t}- \\
&-\sum_{i} \frac{1}{d_{i}}\left(\ddot{R}_{i} R_{i}^{2}+2 R_{i} \dot{R}_{i}^{2}\right), \\
& p_{\text {diff }}(R, t)=\left(P_{0}+\frac{2 \sigma}{R_{0}}\right)\left(R_{0} / R\right)^{3 \gamma}-\frac{2 \sigma}{R}-\frac{4 \mu}{R} \dot{R}-p_{t} .
\end{aligned}
$$

$\sum d_{i}$ can be replaced by the coupling strength of bubbles $S$. The detail of derivation process is referred to Yasui et al. [28]. Instantaneous liquid pressure changing linearly [30] can be expressed as:

$$
p_{t}=p_{v}+\frac{p_{2}-p_{v}}{\tau} t
$$

The pressure recovery time is defined as:

$$
\tau=\frac{L}{\left(v_{0}+v_{p}\right) / 2},
$$

The length scale of eddy is given as:

$$
L=8 d_{p}
$$

The fluid velocity at the orifice can be obtained by:

$$
v_{0}=\sqrt{\frac{2\left(p_{2}-p_{v}\right)}{\rho C_{i}}} .
$$

The fluid velocity at the pipe can be written as:

$$
v_{p}=\left(d_{0} / d_{p}\right)^{2} v_{0}=\beta^{2} v_{0} .
$$

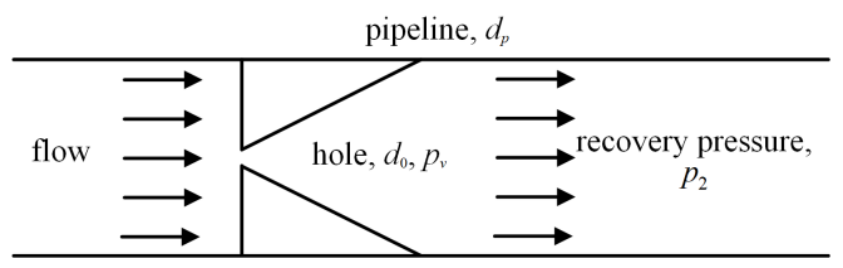

Fig. 1 Geometry for orifice plate

In the present model, the temperature and pressure inside the bubble are approximated as follows [34]:

$$
\begin{aligned}
& T=T_{\infty}\left(\frac{R_{\max }}{R}\right)^{3(\gamma-1)}, \\
& p=\left[p_{v}+\left(P_{0}+\frac{2 \sigma}{R_{0}}\right)\left(\frac{R_{0}}{R_{\max }}\right)^{3}\right]\left(\frac{R_{\max }}{R}\right)^{3 \gamma} .
\end{aligned}
$$

According to analysis in Ref. [34], internal energy inside a bubble neglecting heat transfer between the bubble and the liquid can be obtained by:

$$
\Delta E=\frac{p(t) V(t)-P_{\min } V_{\max }}{\gamma-1} .
$$

The whole activity of interacting bubbles in hydrodynamic cavitation is translated into a differential equation, resolved numerically using 4-5 Runge-Kutta algorithm. The relative and absolute tolerances are $10^{-10}$ and $10^{-11}$, respectively. The initial conditions are $R=R_{0}(t=0 \mathrm{~s}), \mathrm{d} R / \mathrm{d} t=0$ $(t=0 \mathrm{~s})$. The following values are employed in the simulations $[30,31,35-38]: \quad T_{\infty} 293.15 \mathrm{~K} ; \quad P_{0}=$ $=1 \times 10^{5} \mathrm{~Pa} ; p_{2}=1 \times 10^{5} \mathrm{~Pa} ; p_{v}=2330 \mathrm{~Pa} ; \rho=1000 \mathrm{~kg} / \mathrm{m}^{3}$; $\sigma=0.072 \mathrm{~N} / \mathrm{m} ; c=1480 \mathrm{~m} / \mathrm{s} ; \gamma=1.4 ; \mu=1.005 \times 10^{-3} \mathrm{~Pa} \cdot \mathrm{s}$; $C_{i}=0.8 ; \beta=0.5 ; d_{p}=0.03 \mathrm{~m}$. If not specified, the initial bubble radius is $R_{0}=100 \mu \mathrm{m}$ and the coupling strength of bubbles is $S=1000 \mathrm{~m}^{-1}$.

\section{Results and discussions}

In the process of radial oscillations, the maximum 
radius of bubble expansion can reach two times than its initial radius resulting in transient cavitation. During bubble collapse violently, the energy is highly concentrated forming a high temperature and pressure environment inside the bubble. Several chemical reactions are taken place inducing sonochemical effects [14, 21, 34, 37]. In this section, the radial motion and inertial energy of single bubble and single bubble in a cluster containing different number of bubbles are analyzed by the method of numerical simulation.

The calculations of bubble radius and internal energy of single bubble are shown in Fig. 2. With time goes on, the maximum bubble radius decreases and bubble expansion/compression periods are shortened. The expansion and collapse of the bubble are the process of energy gathering and releasing, respectively. During bubble formation and expansion, the energy around the bubble is captured. When the pressure inside the bubble begins to recover, the energy is released. The energy is concentrated during bubble collapse and then pressure pulse is radiated. From Fig. 2, the bubble compresses to the minimum radius at the fifth collapse, which is $68.97 \%$ of its initial radius. The internal energy reaches its maximum value. The pressure and temperature inside the bubble are shown in Fig. 3. Their variation trends are similar to the internal energy. At the fifth collapse, the pressure and temperature increase up to 105.96 atm and $2724.06 \mathrm{~K}$, respectively. It can be seen that the increase the pressure and temperature result in the rise of internal energy during bubble collapse.

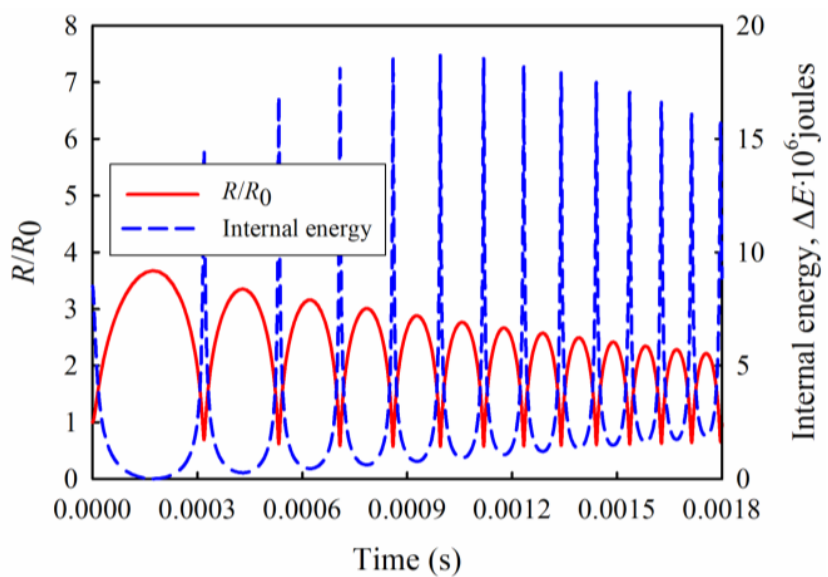

Fig. 2 The normalized bubble radius and internal energy inside a bubble as a function of time

The effects of the coupling strength $S$ in range of $0-100000 \mathrm{~m}^{-1}$ on the bubble dynamics have been studied. Comparisons of the dynamics between single bubble and a bubble in the cluster are shown in Fig. 4. The growing and collapsing behaviors of a bubble under the effects of bubblebubble interactions are similar to that of single bubble. In the case of a fixed $S$, the maximum expansion amplitude decreases and the minimum compression amplitude increases gradually during each bubble expansion/compression period as time goes on.

In any given bubble expansion/compression period, bubble expand less and collapse more strongly as $S$ increasing from 0 to $100000 \mathrm{~m}^{-1}$. The maximum radii of bubble expansion are $3.68,3.64,3.48$ and 3.12 times than their initial radii and the minimum radii of bubble collapse are $57.37 \%$, $55.58 \%, 47.63 \%$ and $36.68 \%$ of their initial radii. The time of bubble expansion to the maximum radius are $172.18 \mu \mathrm{s}$,
$186.45 \mu \mathrm{s}, 276.17 \mu \mathrm{s}$ and $525.13 \mu \mathrm{s}$. The time of bubble collapse to the minimum radius are $146.42 \mu \mathrm{s}, 163.44 \mu \mathrm{s}$, $234.99 \mu$ s and $439.34 \mu$ s. The bubble lifetime increases by $200 \%$ taking bubble-bubble interaction into account ( $S=100000 \mathrm{~m}^{-1}$ ) as compare to the oscillation of a single bubble. Thus, it can be concluded that the bubble-bubble interactions not only weakens the bubble expansion, but also enhances the bubble collapse. The time of bubble reaching the maximum and minimum radii are delayed. The more of bubble numbers are in a cluster, the more significant of the delay effect is. The same conclusions were also obtained in the study of the dynamics of a bubble cluster under acoustic excitation in Ref. [33].

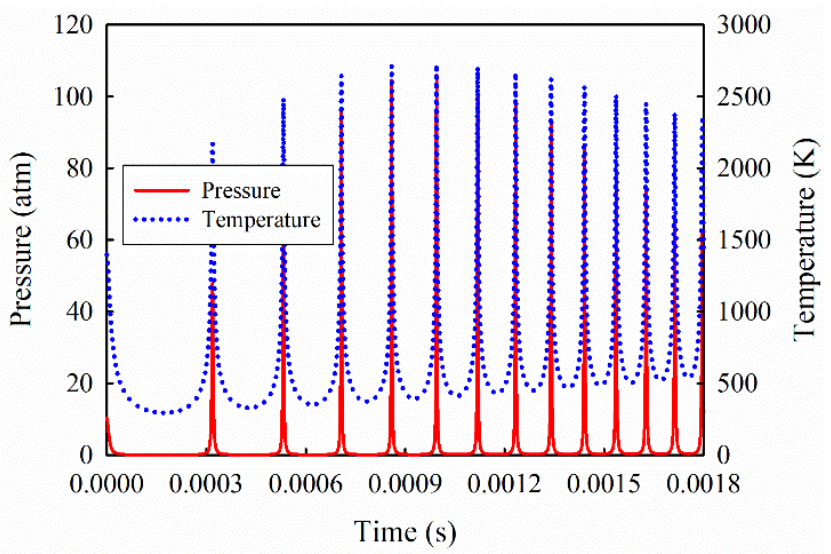

Fig. 3 The pressure and temperature inside a bubble as a function of time

In order to seek a relationship between the internal energy and the number of bubbles in the cluster, numerical simulations have been performed for different $S(0,1000$, 10000 and $100000 \mathrm{~m}^{-1}$ ) as shown in Fig. 5. It has been found that the max internal energy increases as $S$ increases. In other words, the concentration of energy inside a bubble is enhanced with increasing number of adjacent bubbles. This phenomenon is the result of many factors, such as the change of local speed of sound, the increase of temperature, pressure and bubble expansion/compression time. Nevertheless, there are two explanations for this phenomenon based on numerical results: one is the collapse strength of inner bubble is enhanced due to the pressure pulses radiated by the earlier collapse of outer bubbles acting on the inner bubble. From the experimental results in Ref. [20], the shock waves generated by the collapse of outer bubbles affected the inner bubbles due to their delayed collapse. The interactions of a bubble with a shock wave resulting in making the bubble collapse more violently have been proved experimentally [39] and numerically [40]. The other one is that the increase of $S$ makes the bubble expansion/compression periods increase. That is to say the time of bubble expansion and compression increase. The bubble has a stronger ability to gather and release energy. Thus, the increase of $S$ results in a stronger collapse. The following conjecture can be obtained. There exists a smaller number of bubbles in the cluster, the first mechanism dominates due to the expansion ratio and compression ratio having little changes as shown in Fig. 4. Otherwise, the second explanation becomes a major mechanism. 


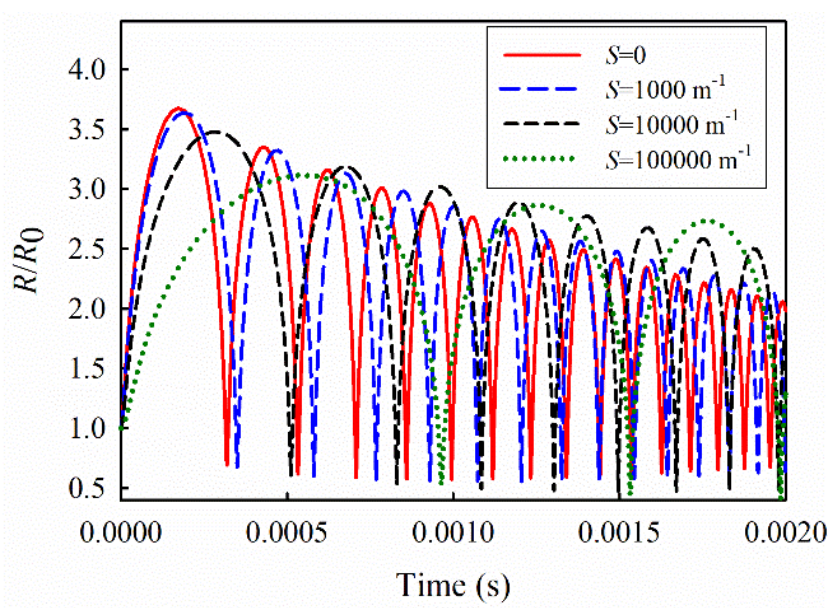

Fig. 4 The normalized bubble radius as a function of time with different interaction strength $S$

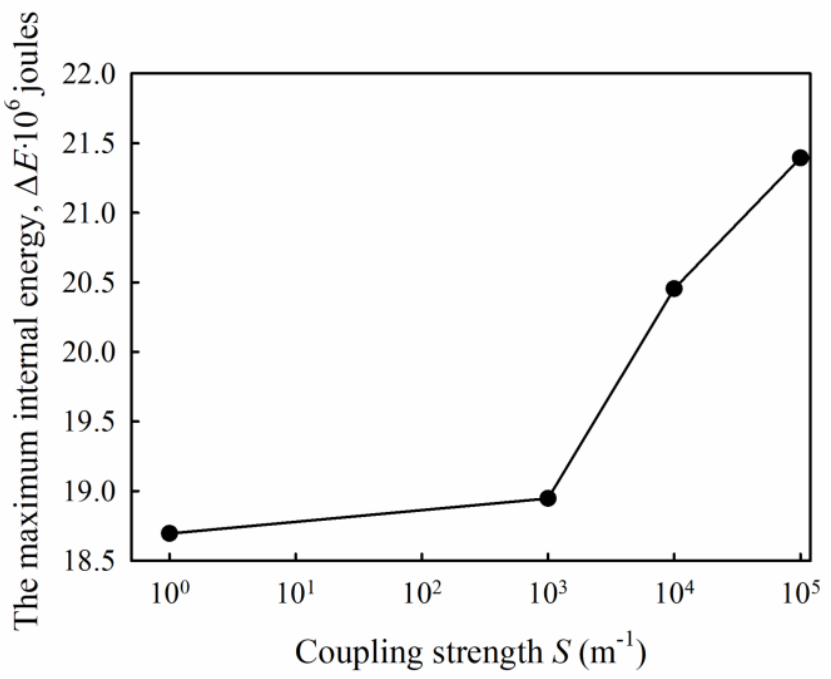

Fig. 5 The maximum internal energy as a function of the coupling strength $S$ of a bubble cloud

The initial radius of hydrodynamic bubble usually varies between $50 \mu \mathrm{m}$ and $500 \mu \mathrm{m}$ depending on flow conditions [35]. The change of the orifice's size, liquid vaporization's degree, the dissolved gas concentration as well as the species in liquid, can all alter the initial bubble radius. $[37,38]$. The initial bubble radius is an important factor affecting the energy concentration and release during bubble growth and collapse, respectively. It can be seen as shown in Fig. 6 that in an increment of the initial bubble radius, both the maximum expansion radius decreases and the minimum collapse radius increases in a small range. However, the time of bubble growing to the maximum radius and shrinking to the minimum radius have a significant increase. For example, the maximum sizes obtained are $3.69 R_{0}$ and $2.95 R_{0}$ for the initial sizes of $50 \mu \mathrm{m}$ and $500 \mu \mathrm{m}$, respectively. The minimum sizes are $0.69 R_{0}$ and $0.53 R_{0}$. While the growth time are $94.41 \mu \mathrm{s}$ and $717.09 \mu \mathrm{s}$, the collapse time are $89.81 \mu \mathrm{s}$ and $519.48 \mu \mathrm{s}$. The amplitudes are increased by nearly $651.61 \%$ and $476.23 \%$. From the works in Refs. [30, 31, 33], the similar conclusions were obtained in the study of the two bubbles interactions in hydrodynamic cavitation and the bubble cloud dynamics in acoustic cavitation.

The maximum internal energy of the bubble is a function of initial bubble radius displayed in Fig. 7. The bubble absorbs energy from the surrounding liquid to keep the bubble oscillating. When bubble collapse to the minimum value, the internal energy inside the bubble rise to the maximum value and pressure pulses are radiated outward. When initial radius of the bubble increases, the maximum internal energy of the bubble increases monotonically and the magnitude of the increase has a substantial rise. The maximum internal energy of a bubble with an initial radius of $500 \mu \mathrm{m}$ is 3 orders of magnitude more than that of a bubble with an initial radius of $50 \mu \mathrm{m}$. As can be seen from the result in Ref. [30], the larger of initial bubble radius is, the larger of the peak value of pressure pulse is. Experiments on $\mathrm{OH}^{-}$radicals detecting, disinfection and the degradation of substances with different volatility indicated hydrodynamic cavitation working as a low-frequency ultrasonic generator [41]. In the study of acoustic cavitation, it has been found that formed bubble are larger and they are forced to collapse more aggressively with low ultrasonic frequencies. More energy releases in terms of pressure pulses [42].

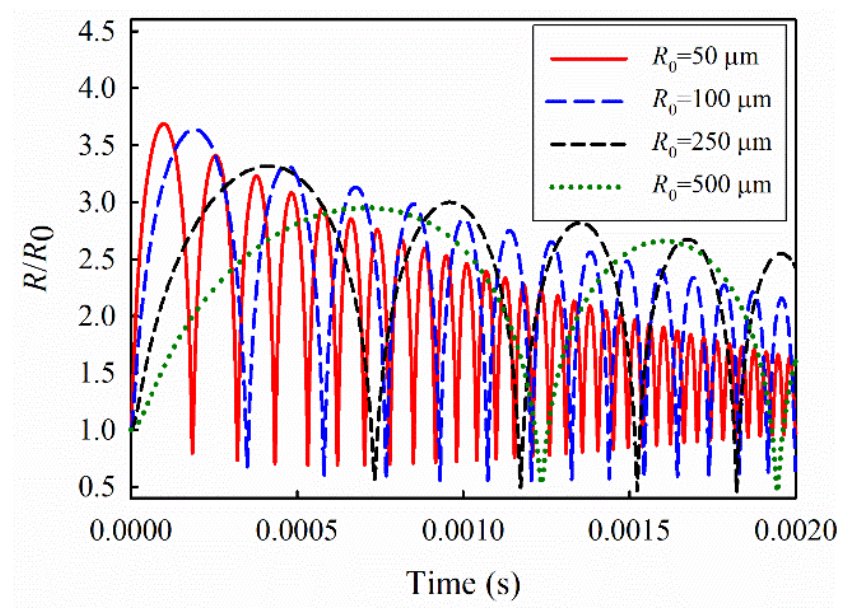

Fig. 6 The normalized bubble radius as a function of time with different initial bubble radius $R_{0}$

Recovery pressure can be altered by changing the inlet pressure and the design of the orifice [36, 41]. The effects of recovery pressure of orifice plate on the bubble oscillations and the maximum internal energy inside the bubble are shown in Fig. 8 and 9. From Fig. 8, it can be seen that with the increase of final recovery pressure $\left(p_{2}=1 \times 10^{5}\right.$ $\mathrm{Pa}, 2 \times 10^{5} \mathrm{~Pa}$ and $3 \times 10^{5} \mathrm{~Pa}$ ), expansion/ compression ratio $\left(R_{\max } / R_{\min }\right)$ are $6.55,6.54$ and 6.25 , respectively. The time length of bubble compression to the minimum radius is greatly reduced, which are $186.46 \mu$ s, $145.99 \mu$ s and 107.75 $\mu \mathrm{s}$. The maximum velocities of the bubble collapse reach $15.05 \mathrm{~m} / \mathrm{s}, 19.28 \mathrm{~m} / \mathrm{s}$ and $20.94 \mathrm{~m} / \mathrm{s}$. The rise of recovery pressure causing the pressure gradients to add in liquid and then the bubble collapse more intensively. This is consistent with the results of the single bubble studies in hydrodynamic cavitation. The increase of recovery pressure makes bubble collapse more violently, and the stronger pressure pulses could be formed [38]. The chemical reactions during bubble oscillations could be optimized by a fast recovery pressure combined with a moderate bubble density [41]. From the analysis in Ref. [37], recovery pressure and ultrasound intensity were analogous parameters in hydrodynamic and acoustic cavitation. Merouani et al. [34] revealed the powers during bubble oscillations were higher at large excitation 
amplitude in acoustic cavitation for all ultrasound frequencies. With a rise in final recovery pressure, the increase of the maximum internal energy is shown in Fig. 9. Krishnan et al. [43] revealed that with the increase of recovery pressure the maximum of temperature and pressure increased during bubble collapse, and more water vapor got out of the bubble. More $\mathrm{OH}^{-}$radicals were produced under the conditions of high temperature and pressure.

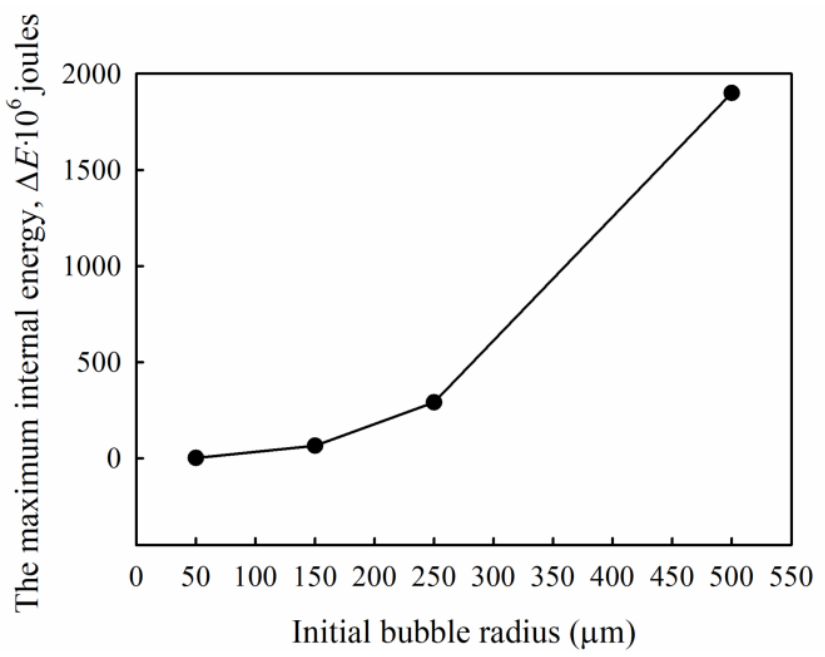

Fig. 7 The maximum internal energy as a function of initial bubble radius

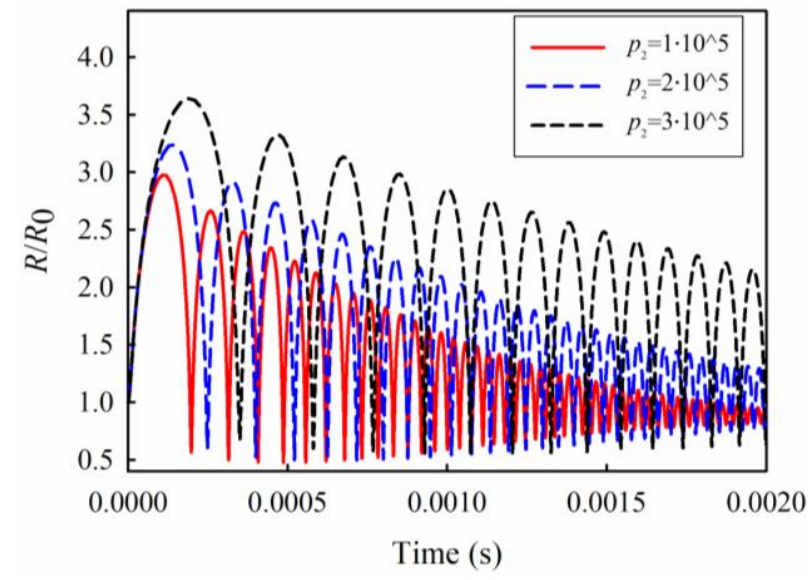

Fig. 8 The normalized bubble radius as a function of time with different recovery pressure $p_{2}$.

The effect of bubble-bubble interaction on oscillation of the bubble has been investigated both theoretically and experimentally by many researchers. Nevertheless, many of them are on two bubbles [6, 16-18, 22-24]. Furthermore, in most of the previous studies, the method of bubble pulsation is not by hydrodynamic pressure variation but by energy focus [7, 8, 16-19], pressure pulses [20] or ultrasound [6, 21-29]. Therefore, in the present analysis, the pulsation of a bubble has been studied theoretically under hydrodynamic pressure variation taking bubble-bubble interactions into account. The instantaneous pressure in liquid at any time is obtained assuming linear variation between the pressure at contraction section and recovery pressure at downstream of the orifice or turbulent fluctuation. It can be found from researchers' analysis the variation trends of bubble dynamics were consistent under the action of the instantaneous pressure obtained by these two methods [30, 31, 35$38,44]$. Hence, the turbulent fluctuation is not introduced into the model accounting for bubble-bubble interactions in hydrodynamic cavitation. In the case of hydrodynamic cavitation, bubble dynamics, i.e. radial motion and internal energy, are influenced by the number of bubbles in a cluster (i.e. bubble cluster size), the initial bubble size and recovery pressure. Therefore, this paper can provide references for the operation and design of hydrodynamic cavitation system.

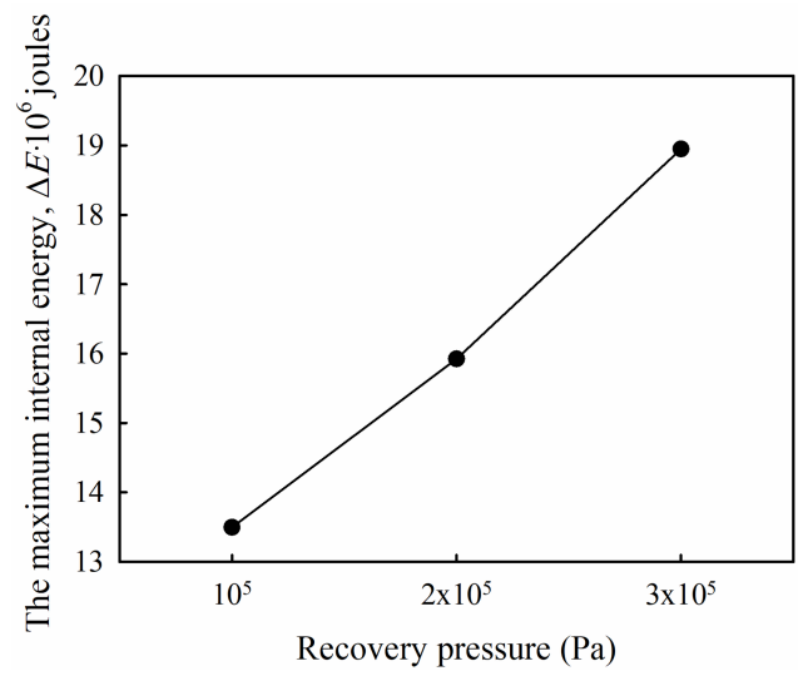

Fig. 9 The maximum internal energy as a function of recovery pressure

\section{Conclusions}

A model describing bubble-bubble interactions in hydrodynamic cavitation has been presented in this paper. The radial oscillations and internal energy have been numerically investigated for various conditions of the coupling strength of bubbles, initial bubble radius and recovery pressure to explore the key parameters on dynamics of the bubble cluster. The results presented in this paper can provide references for the design and operation to maximize the cavitation effects in hydrodynamic cavitation reactor. The main findings are as follows.

1. Due to the effect of bubble-bubble interaction, the bubble lifetime increases (e.g. an increase of $200 \%$ for $S=100000 \mathrm{~m}^{-1}$ ) compared with the oscillation of a single bubble. With the rise of the initial bubble radius, the bubble oscillation time is enhanced. Bubble lifetime for $R_{0}=500 \mu \mathrm{m}$ increases by $566.57 \%$ compared with that for $R_{0}=100 \mu \mathrm{m}$. Therefore, the bubble has enough time to gather and release energy. The maximal internal energy increases, and cavitation strength is also accelerated.

2 . Two possible explanations are given for the reason of the maximum internal energy increasing with the coupling strength. For the smaller number of bubbles in a cluster, the pressure pulses radiated by the earlier collapse of outer bubbles acting on the inner bubble enhances collapse strength. For the larger number of bubbles in a cluster, the bubble expansion/compression periods increase sharply resulting in increasing the abilities of energy concentration and release.

3 . A rise in recovery pressure results in the maximum velocities of the bubble collapse increasing (e.g. $15.05 \mathrm{~m} / \mathrm{s}$ for $p_{2}=1 \times 10^{5} \mathrm{~Pa}, 19.28 \mathrm{~m} / \mathrm{s}$ for $p_{2}=2 \times 10^{5} \mathrm{~Pa}$ and $20.94 \mathrm{~m} / \mathrm{s}$ for $p_{2}=3 \times 10^{5} \mathrm{~Pa}$ ), and the active cavitation intensity of the downstream of the orifice is enhanced. 


\section{References}

1. Blake, J. R.; Gibson, D. C. 1987. Cavitation bubbles near boundaries, Annual Review of Fluid Mechanics 19: 99-123.

https://doi.org/10.1146/annurev.fl.19.010187.000531.

2. Brennen, C. E. 1995. Cavitation and bubble dynamics, New York: Oxford University Press: 15-136. https://doi.org/10.1017/cbo9781107338760.004.

3. Lauterborn, W.; Kurz, T. 2010. Physics of bubble oscillations, Reports on Progress in Physics 73(10): 106501-1-88. https://doi.org/10.1088/0034-4885/73/10/106501.

4. Wang, Q. X.; Blake, J. R. 2010. Non-spherical bubble dynamics in a compressible liquid. Part 1. Travelling acoustic wave, Journal of Fluid Mechanics 659: 191-224. https://doi.org/10.1017/s0022112010002430.

5. Wang, Q. X.; Blake, J. R. 2011. Non-spherical bubble dynamics in a compressible liquid. Part 2. Acoustic standing wave, Journal of Fluid Mechanics 679: 559581. https://doi.org/10.1017/jfm.2011.149.

6. Zhang, Y. N.; Zhang, Y. N.; Li S. C. 2016. The secondary Bjerknes force between two gas bubbles under dualfrequency acoustic excitation, Ultrasonics Sonochemistry (29), 129-145.

https://doi.org/10.1016/j.ultsonch.2015.08.022.

7. Zhang, Y. N.; Chen, F. P.; Zhang, Y. N.; et al. 2018. Experimental investigations of interactions between a laser-induced cavitation bubble and a spherical particle, Experimental Thermal and Fluid Science 98:645-661. https://doi.org/10.1115/1.861851_ch82.

8. Lv, L.; Zhang, Y. X.; Zhang, Y. N.; et al. 2019. Experimental investigations of the particle motions induced by a laser-generated cavitation bubble, Ultrasonics Sonochemistry 56: 63-76.

https://doi.org/10.1016/j.ultsonch.2019.03.019.

9. Huang, R. F.; Ji, B.; Luo, X. W.; et al. 2015. Numerical investigation of cavitation-vortex interaction in a mixedflow waterjet pump, Journal of Mechanical Science and Technology 29(9): 3707-3716. https://doi.org/10.1007/s12206-015-0816-4.

10. Wang, C.; Zhang, Y. X.; Hou, H. C.; et al. 2019. Entropy production diagnostic analysis of energy consumption for cavitation flow in a two-stage LNG cryogenic submerged pump, International Journal of Heat and Mass Transfer 129:342-356.

https://doi.org/10.1016/j.ijheatmasstransfer.2018.09.070.

11. Zhang, Y. N.; Zhang, Y. N.; Qian, Z. D.; et al. 2016. A review of microscopic interactions between cavitation bubbles and particles in silt-laden flow, Renewable and Sustainable Energy Reviews 56: 303-318. https://doi.org/10.1016/j.rser.2015.11.052.

12. Mason, T. J. 2016. Ultrasonic cleaning: An historical perspective, Ultrasonics Sonochemistry 29: 519-523. https://doi.org/10.1016/j.ultsonch.2015.05.004.

13. An, Y. 2011. Formulation of multibubble cavitation, Physical Review E 83(6Part2): 066313. https://doi.org/10.1103/physreve.83.066313.

14. Pokhrel, N.; Vabbina, P. K; Pala, N. 2016. Sonochemistry: Science and Engineering, Ultrasonics Sonochemistry, 29: 104-128. https://doi.org/10.1016/j.ultsonch.2015.07.023.

15. Petkovšek, M.; Zupanc, M.; Dular, M.; et al. 2013. Rotation generator of hydrodynamic cavitation for water treatment, Separation and Purification Technology 118: 415-423. https://doi.org/10.1016/j.seppur.2013.07.029.

16. Fong, S. W.; Adhikari, D.; Klaseboer, E.; et al. 2009. Interactions of multiple spark-generated bubbles with phase differences, Experiments in Fluids 46(4): 705-724. https://doi.org/10.1007/s00348-008-0603-4.

17. Chew, L. W.; Klaseboer, E.; Ohl C. D.; et al. 2011. Interaction of two differently sized oscillating bubbles in a free field, Physical Review E 84: 066307. https://doi.org/10.1007/s00348-008-0603-4.

18. Han, B.; Köhler, K.; Jungnickel, K.; et al. 2015. Dynamics of laser-induced bubble pairs, Journal of Fluid Mechanics 771: 706-742. https://doi.org/10.1007/s00348-008-0603-4.

19. Cui, P.; Wang, Q. X.; Wang, S. W.; et al. 2016. Experimental study on interaction and coalescence of synchronized multiple bubbles, Physics of Fluids 28: 012103. https://doi.org/10.1063/1.4939007.

20. Bremond, N.; Arora, M.; Ohl, C. D.; et al. 2006. Controlled Multibubble Surface Cavitation, Physical Review Letters 96(22): 224501. https://doi.org/10.1103/physrevlett.96.224501.

21. Stricker, L.; Dollet, B.; Rivas, D. F. 2013. Interacting bubble clouds and their sonochemical production, Journal of the Acoustical Society of America 134(3): 18541862. https://doi.org/10.1121/1.4816412.

22. Ida, M. 2003. Alternative interpretation of the sign reversal of secondary Bjerknes force acting between two pulsating gas bubbles, Physical Review E 67(5 Part2): 056617. https://doi.org/10.1103/physreve.67.056617.

23. Sugita, N.; Sugiura, T. 2017. Nonlinear normal modes and localization in two bubble oscillators, Ultrasonics 74 : 174-185. https://doi.org/10.1016/j.ultras.2016.10.008.

24. Valier-Brasier, T.; Conoir, J. M. 2019. Resonant acoustic scattering by two spherical bubbles, Journal of the Acoustical Society of America 145(1): 301-311. https://doi.org/10.1121/1.5087556.

25. Nasibullaeva, E. S.; Akhatov, I. S. 2013. Bubble cluster dynamics in an acoustic field, Journal of the Acoustical Society of America 133(6): 3727-3738. https://doi.org/10.21662/uim2008.1.004.

26. Fuster, D.; Colonius, T. 2011. Modelling bubble clusters in compressible liquids, Journal of Fluid Mechanics 688: 352-389. https://doi.org/10.1017/jfm.2011.380.

27. Fuster, D.; Conoir, J .M.; Colonius, T. 2014. Effect of direct bubble-bubble interactions on linear-wave propagation in bubbly liquids, Physical Review E 90(6): 063010. https://doi.org/10.1017/jfm.2011.380.

28. Yasui, K.; Iida, Y.; Tuziuti, T.; et al. 2008. Strongly interacting bubbles under an ultrasonic horn, Physical Review E 77: 016609. https://doi.org/10.1017/jfm.2011.380.

29. Yasui, K.; Lee, J.; Tuziuti, T.; et al. 2009. Influence of the bubble-bubble interaction on destruction of encapsulated microbubbles under ultrasound, Journal of the 
Acoustical Society of America 126(3): 973-982. https://doi.org/10.21662/uim2008.1.004.

30. Li, F. Ch.; Cai, J.; Huai, X. L.; et al. 2013. Interaction mechanism of double bubbles in hydrodynamic cavitation, Journal of Thermal Science 22(3): 242-249. https://doi.org/10.1007/s11630-013-0619-9.

31. Lv, L.; Zhang, Y. X.; Zhang, Y. N. 2019. The effect of turbulence on the dynamics of two bubbles in hydrodynamic cavitation, IOP Conference Series: Earth and Environmental Science, 240(6): 1-8. https://doi.org/10.1088/1755-1315/240/6/062042.

32. Keller, J. B.; Miksis, M. 1980. Bubble oscillations of large amplitude, Journal of the Acoustical Society of America 68(2): 628-633. https://doi.org/10.21236/ada095754.

33. Yasui, K.; Towata, A.; Tuziuti, T.; et al. 2011. Effect of static pressure on acoustic energy radiated by cavitation bubbles in viscous liquids under ultrasound, Journal of the Acoustical Society of America 130(5): 3233-3242. https://doi.org/10.1121/1.3626130.

34. Merouani, S.; Hamdaoui, O.; Rezgui, Y.; et al. 2014. Energy analysis during acoustic bubble oscillations: Relationship between bubble energy and sonochemical parameters, Ultrasonics 54(1): 227-232. https://doi.org/10.1016/j.ultras.2013.04.014.

35. Kumar, P.; Khanna, S.; Moholkar, V. S. 2012. Flow regime maps and optimization thereby of hydrodynamic cavitation reactors, AIChE Journal 58(12): 3858-3866. https://doi.org/10.1002/aic.1377.

36. Moholkar, V. S.; Pandit, A. B. 1997. Bubble behavior in hydrodynamic cavitation: effect of turbulence, AIChE Journal 43(6):1641-1648. https://doi.org/10.1002/aic.690430628

37. Moholkar, V. S.; Kumar, P. S.; Pandit, A. B. 1999. Hydrodynamic cavitation for sonochemical effects, Ultrasonics Sonochemistry 6(1-2): 53-65. https://doi.org/10.1016/s1350-4177(98)00030-3.

38. Gogate, P. R.; Pandit, A. B. 2000. Engineering design methods for cavitation reactors II: hydrodynamic cavitation, AIChE Journal 46(8):1641-1649. https://doi.org/10.1002/aic.690460815.

39. Sankin, G. N.; Simmons, W. N.; Zhu S. l.; et al. 2005. Shock wave interaction with laser-generated single bubbles. Physical Review Letters 95:034501. https://doi.org/10.1063/1.2210367.

40. Betney, M. R.; Tully, B.; Hawker, A.; et al. 2015. Computational modelling of the interaction of shock waves with multiple gas-filled bubbles in a liquid, Physics of Fluids 27(3): 036101. https://doi.org/10.1063/1.4914133.

41. Arrojo, S.; Benito, Y.; Tarifa, M. 2008. A parametrical study of disinfection with hydrodynamic cavitation, Ultrasonics Sonochemistry 15: 903-908. https://doi.org/10.1016/j.ultsonch.2007.11.001.

42. Mason, T. Y.; Cobley, A. J.; Graves, J. E.; et al. 2011. New evidence for the inverse dependence of mechanical and chemical effects on the frequency of ultrasound, Ultrasonics Sonochemistry 18(1): 226-230. https://doi.org/10.1016/j.ultsonch.2010.05.008

43. Krishnan, J. S.; Dwivedi, P.; Moholkar, V. S. 2006. Numerical investigation into the chemistry induced by hydrodynamic cavitation, Industrial and Engineering Chemistry Research 45(4): 1493-1504. https://doi.org/10.1021/ie050839t.

44. Moholkar, V. S. 2016. Mathematical Models for Sonochemical Effects Induced by Hydrodynamic Cavitation, Handbook of Ultrasonics and Sonochemistry 1-48. https://doi.org/10.1007/978-981-287-470-2 51-1.

\section{Lv, X. Luo, H. X. Zhang, B. Cui, L. H. Chen}

\section{THE NUMERICAL INVESTIGATION ON BUBBLE INTERACTION DYNAMICS IN HYDRODYNAMIC CAVITATION}

S u m m a r y

Bubble-bubble interactions are of great importance for bubble dynamics. A mathematical model describing the dynamics of a bubble cluster in hydrodynamic cavitation is presented. The interaction strength (i.e. the number density of bubbles) is introduced into Keller-Misis equation. Using this model, numerical investigations of bubble dynamics (i.e radial motion and internal energy) of single bubble in a cluster containing high number density of bubbles have been carried out due to linear pressure gradient. With the increase of interaction strength, the time of bubble reaching the maximum and minimum radii are delayed. The more of bubbles are in a cluster, the more significant of the delay effect is. The maximum internal energy inside the bubble is closely related to interaction strength (i.e. positive correlation). Furthermore, the effects of initial bubble radius and recovery pressure of the orifice on bubble dynamics are quantitatively discussed. Based on numerical results, some references are put forward for structure optimization and manipulate of hydrodynamic cavitation reactor.

Keywords: bubble-bubble interactions, hydrodynamic cavitation, bubble dynamics, interaction strength, radial motion, internal energy.

Received June 15, 2020

Accepted April 07, 2021

This article is an Open Access article distributed under the terms and conditions of the Creative Commons Attribution 4.0 (CC BY 4.0) License (http://creativecommons.org/licenses/by/4.0/). 